\title{
Underground versatile laser scanning solution
}

\author{
G Rodriguez Maptek, Canada
}

\begin{abstract}
Laser scanning and 3D point cloud data is used extensively in open pit and underground mining operations. Point cloud data generated with laser scanners has improved survey, conformance, and geotechnical process efficiency and has become a key data component for planning, operations and decision-making.

The advent of new software tools designed specifically to manage real-time 3D scan data has also improved the accuracy, quality and usability of underground scan data, revolutionising workflows through the industry.

In this paper, several case studies will demonstrate the versatility and value of laser scanning in the context of underground operations demonstrating the use of laser scanning and derived point cloud data to improve efficiency, reduce costs and increase the safety of staff and assets across the operation.
\end{abstract}

Keywords: laser scanning, filtering, modelling, productivity

\section{Introduction}

For more than 15 years, laser scanning technology has driven transformation in both open pit and underground mining industries. The main benefits of laser scanning are speed, precision and security in the data capture. Numerous recent hardware and software changes make scanning an efficient and intuitive process. Efficient workflows generate economic value for the end user and mining companies. Increased quantity and quality of information, coupled with intelligent software algorithms for processing, allow for progressively more efficient processes.

Laser scanning began as a simple tool for capturing topography. Today, laser scans are used by geologists, mining engineers, and geotechnical engineers. Some applications include design compliance, geological mapping, monitoring, geotechnical analysis and volumetric control. New underground scanning hardware improves the field scan workflow and data processing in the software.

This paper presents several underground applications of laser scanning technology. Technical aspects about the workflow to capture data, recording options, filtering and data modelling are explained. The paper also correlates laser output to value for mining companies.

\section{Registration}

The registration process correlates scan data to mine coordinate systems. Registration can use some field control points while the data is collected, however there are also several software tools that can register free scans against fixed reference data. Field and software registration tools can be combined to optimise the scanning operation. The following sections discuss several methods used in the field to collect scan data.

\subsection{Backsight coordinates}

A total station can be used in conjunction with the scanner to generate geo-referenced data. A prism is attached on top of the scanner, and the coordinate of the scanner location is measured from the total station. The red laser pointer in the scanner is used to align the instrument to a known backsight coordinate, which is typically a point on a nearby wall. 


\subsection{Resection}

The scanner can utilise three points with known coordinates to create geo-referenced points. A database of known points loaded into a field tablet is helpful when using the resection method.

\section{$2.3 \quad$ Free scans}

The software allows for import of point data without coordinates, which are registered against existing scans which contain coordinates. When registering free scans, information about scanner location is required. The end user calls on field experience to recognise the level of overlapping needed to align the new and existing scans.

The free scan registration process allows for a short time in the field. One $360^{\circ}$ scan can be performed in about three minutes, which is convenient for quick scans after scaling a round. The quick turnaround time for the scan limits disruption to the mining cycle.

The end user can manually move scans to approximate the alignment of a free scan to an existing scan that includes coordinates.

With one click, automatic initial registration automatically aligns a free scan to an existing scan which includes coordinate data.

Global registration accurately aligns a free scan against a reference scan which contains coordinates. Global registration works best when point sets contain common features and strategic overlaps.

\section{Drift modelling}

There are two primary methods used to model underground drifts. Either method is an acceptable initial processing step for the examples listed later in this paper. The method used often depends on whether there are scanned elements inside the tunnel that should be removed during the modelling process. The term 'elements' refers to installed services inside the tunnel, such as ventilation tubes, pipes or cables. Elements can include temporary objects such as vehicles, trucks or people.

\subsection{Remove unwanted elements}

This refers to the creation of sections to remove scanned elements from the tunnel during the modelling process. The option that generates sections can ignore the points that construct unwanted elements to create a polygon which represents the surface of the drift (Figure 1). Areas with no points on the surface of the drift are extrapolated from existing point data. Multiple polygons are created along the tunnel at a specified distance. The polygons are used to generate a triangulation model.

Additional options may be required to model and connect intersections of multiple tunnels into a single model.

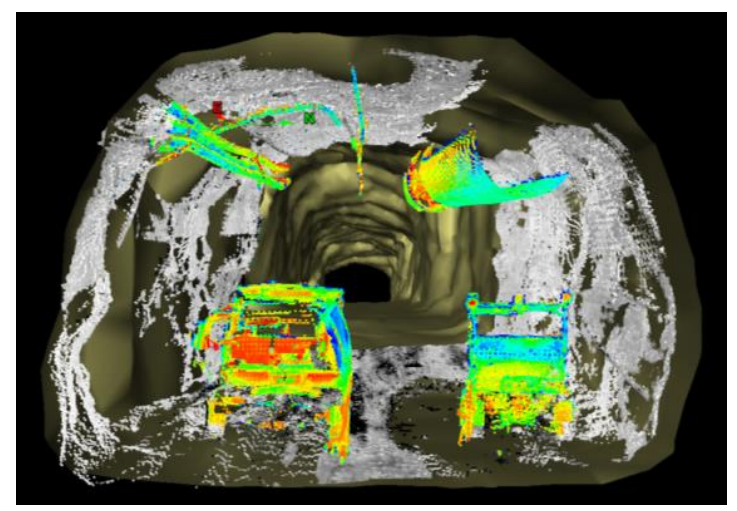

Figure 1 Tunnel model does not include unwanted elements. The tunnel model is yellow. Unwanted elements, which were removed, are shown here in multiple colours 


\subsection{No unwanted elements}

Clean drifts, such as those which were recently mined, may not contain unwanted elements (Figure 2(a)). The process to model this shape is a one-click option which only requires the input of a point distance to build the triangulation without need of user-defined orientation (Figure 2(b)).

The level of detail is determined by the end user. For example, if there is a restriction on the final file size, then the end user may choose to run a minimum separation filter to reduce the density of the point cloud uniformly prior to modelling.

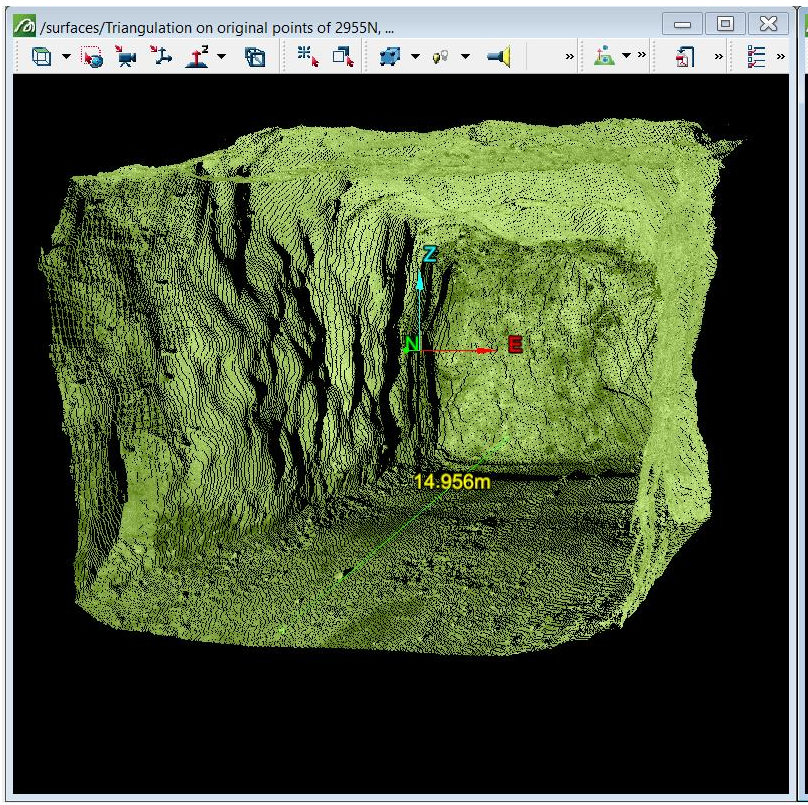

(a)

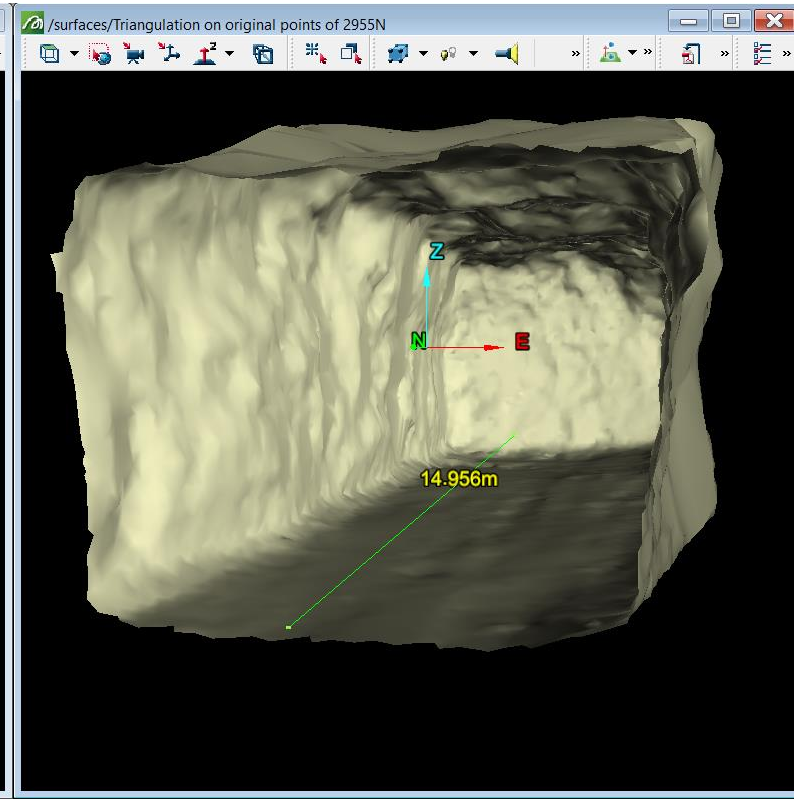

(b)

Figure 2 Clean tunnel modelled with a single click. (a) Point cloud; and, (b) The final model

\section{$4 \quad$ Examples}

\subsection{Tunnel clearance}

The transfer of heavy equipment for operation in different points of the mine has an associated cost. Although the transfer may seem like a simple routine task, certain circumstances may cause unexpected and considerable delays. A clearance analysis may ensure that the trucks will fit along the route of travel, thereby avoiding costly delays and maximising equipment utilisation time.

A few reasons why tunnel clearance evaluation is needed are:

- Opening of a new route for new projects.

- Incorporation of new equipment into the fleet which has different dimensions than the existing equipment.

- Need to bring special heavy equipment to certain sectors of the mine.

- Autonomous equipment.

For example, if a new truck with new dimensions has been incorporated into the fleet, how do we know if the truck will have enough space to move throughout the planned route without having interference? What happens if the truck must stop and return because it does not fit through the route to the production area? Failure to consider clearance along the equipment transportation route can produce delays which may significantly affect the project Gantt chart or mine production. The solution is an accurate 3D scan 
measurement and clearance analysis of the route to make sure the truck will fit properly. A 3D analysis will help identify potential sectors that are subject to modification along the route and therefore to plan and execute an appropriate clearance in advance.

Depending on the tunnels, we can find two possible types of interference for a vehicle moving through the tunnels:

- Existing services such as ventilation ducts, pipes or cables.

- The tunnel section itself. Certain parts may be too narrow or too low, thus preventing passage of equipment with certain dimensions.

The present example shows a reduced portion of a tunnel along the destination route of truck movement. For this purpose, the installed services, such as ventilation tubes, will be temporarily removed to allow clearance for truck transport. The first objective of the job was to scan the tunnel with the services included, but model the tunnel without services. The second goal was to determine if the tunnel section (walls and roof) provided enough clearance along the $370 \mathrm{~m}$ route to accommodate the truck. The modelling method described in Section 3.2 was used to generate a 3D model of the tunnel without services.

To model the path of the truck, the measurements of the silhouette of the truck are needed as input. A catalogue of the truck specifications was used to determine the main measurements. The silhouette of the truck was initially drawn in the software as a single polygon in 2D. The silhouette polygon was expanded with an offset distance of $80 \mathrm{~cm}$ from the original. The bottom of the silhouette polygon was not expanded because the bottom of the polygon represents contact between the truck and the ground. The extended silhouette, shown in yellow in Figure 3, was determined to be a minimum safety distance due the minimum precision while driving and to absorb the turning radius on the curves. The safety distance was provided by the mine.

Using another tool in the software, the silhouette image from the digital catalogue, shown in red in Figure 3, was draped over the triangulation of the truck polygon to provide more visual understanding.

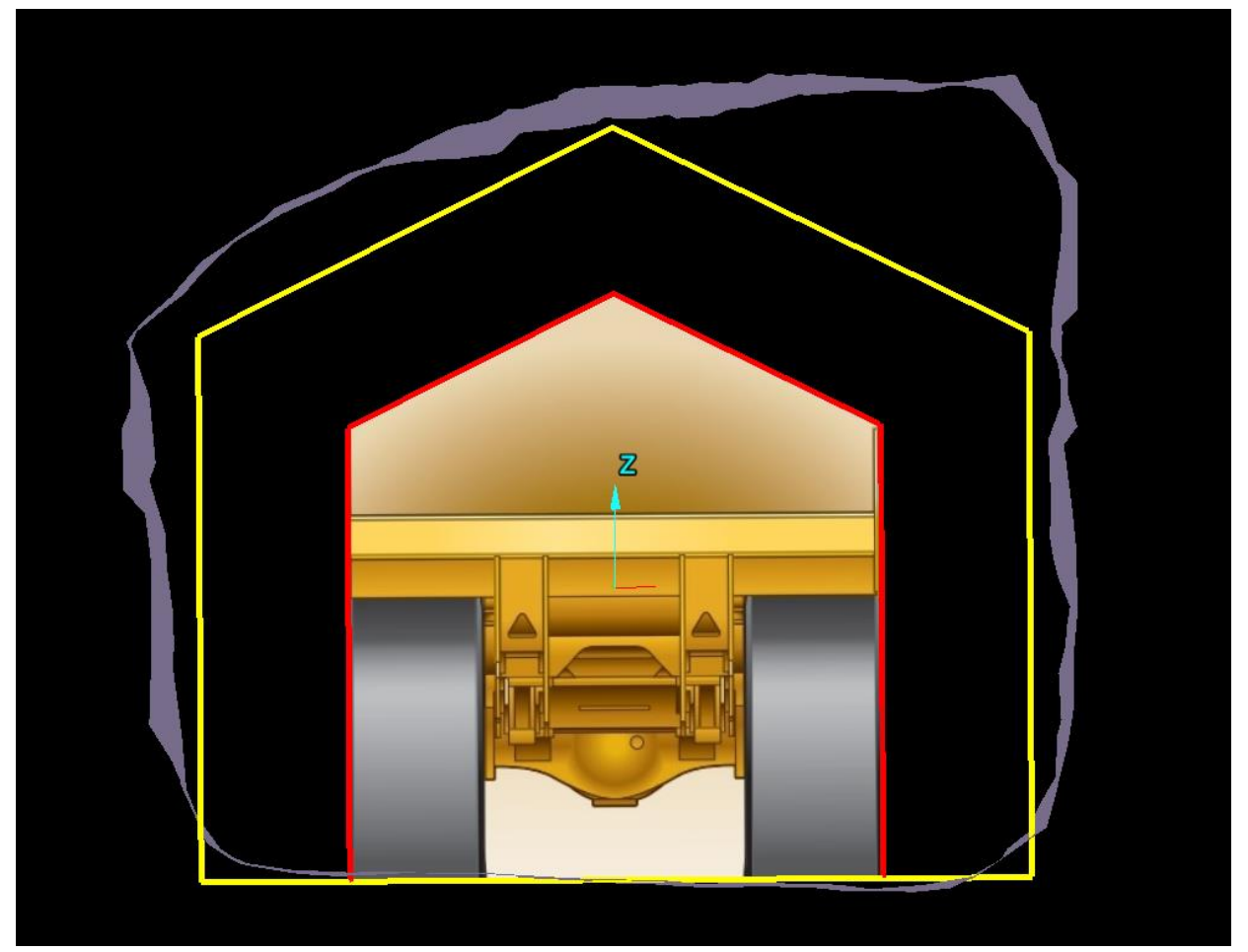

Figure 3 Section view of the silhouette of the truck. The expanded section is in yellow and the actual surface of the tunnel is grey 
The centre line of the route must be drawn manually in plan view along the tunnel direction. The centre line is important as it will serve as a guide to project the silhouette of the truck. When the centre line is finished, the truck silhouette is copied multiple times along the line so the truck is projected along the tunnel route.

The silhouettes are computer-aided design (CAD) polygons that can be modelled with loop triangulation, which is a solid projection that simulates the truck trajectory. The loop triangulation is shown in green in Figure 4.

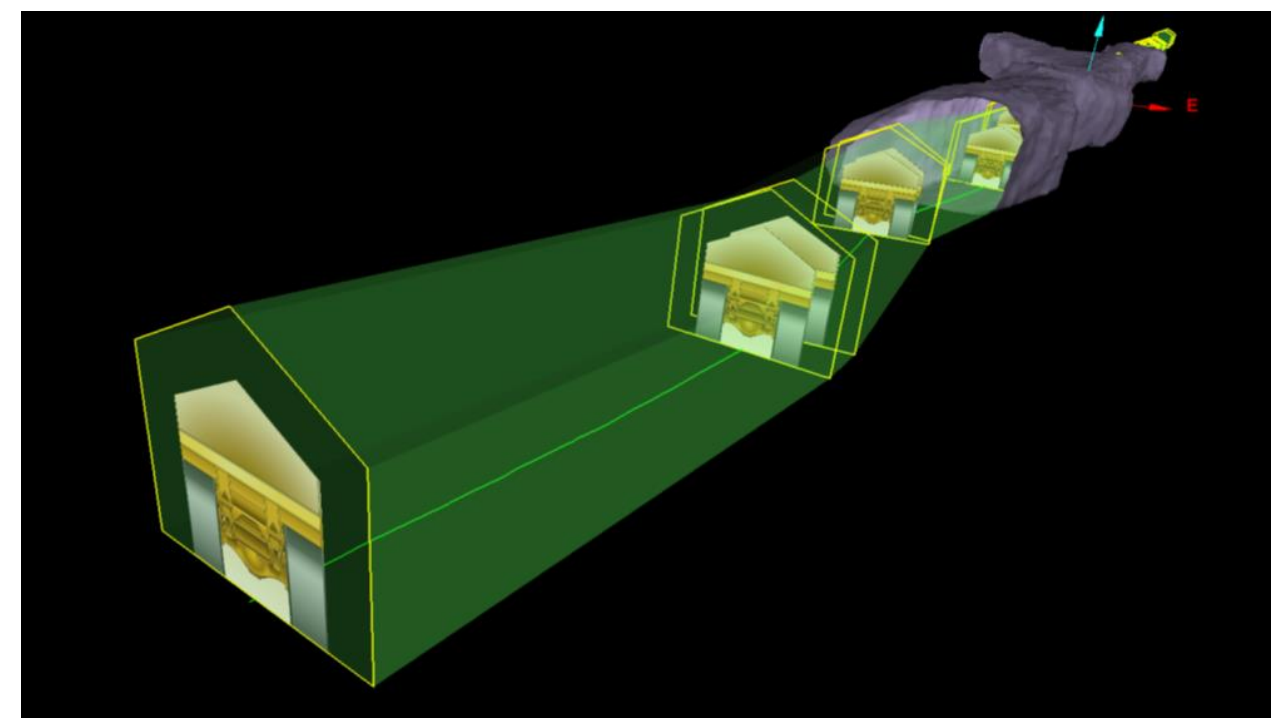

Figure 4 Repeated truck shapes are projected through the centre line

The software is used to run a complex Boolean algorithm on the tunnel model and truck trajectory model. The Boolean tool returns the common volume of two or more input solids. Intersection locations of all input triangulations are analysed in three dimensions to determine multiple points which will define the edge of shared volume. Triangles are reshaped to result in a minimum of three resulting solids with unique volumes. The new intersected solid can be queried for cut and fill volumes, or exploded to get volumes for each sub-solid that was created (Figure 5). All this is done in seconds with one click.

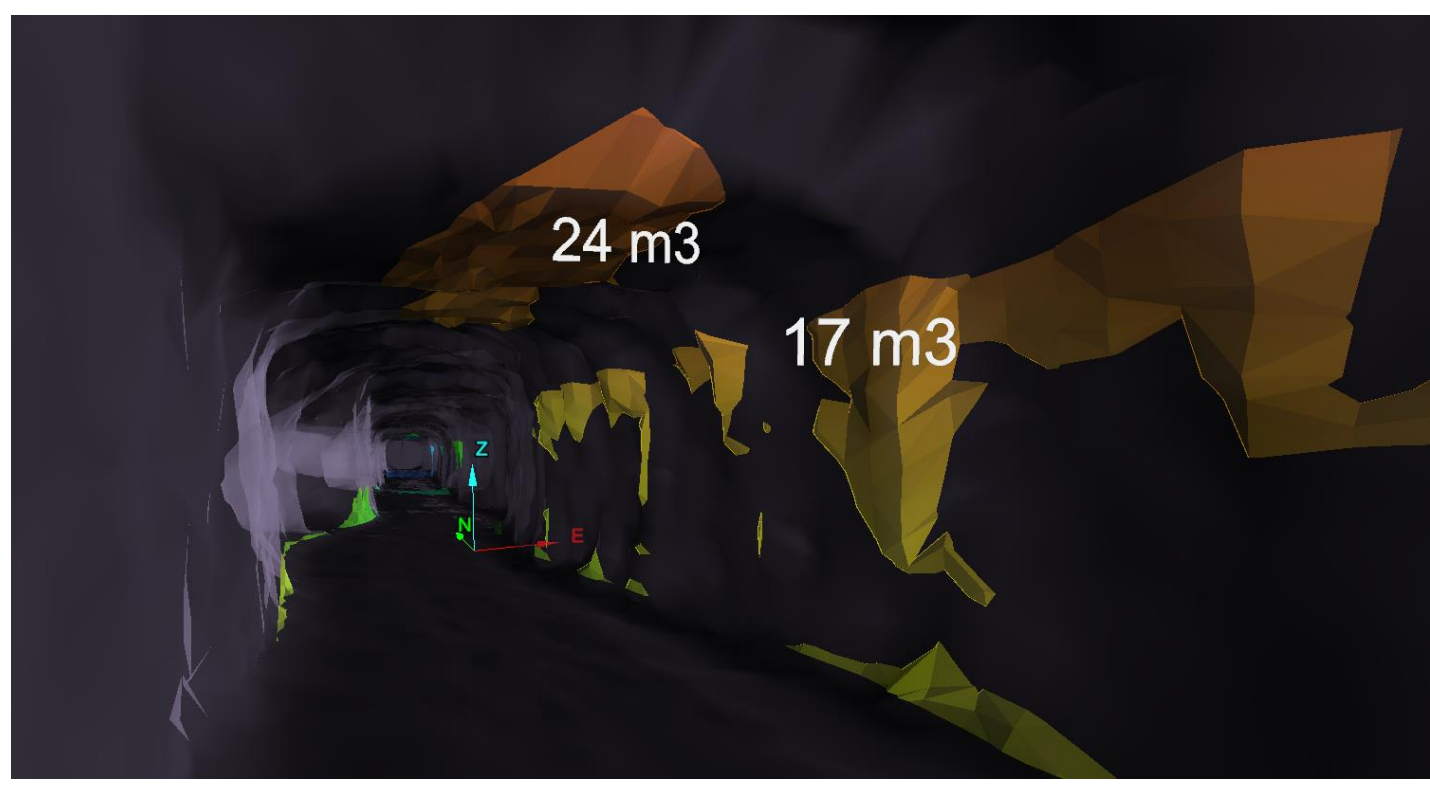

Figure 5 Tunnel model with solids and volumes to dig 
A great addition in this process is the possibility to heat map colour the tunnel based on the distance from the truck trajectory solid. The colours are pre-defined intervals that represent different ranges from one surface to the other.

The final output of this scanning work helped to determine 12 critical points along the route that required more clearance and identified $126 \mathrm{~m}^{3}$ that required excavation. The scanning work plus the processing took only two and a half days and provided accurate information for the decision-making process. It helped to appropriately re-schedule the activities and avoid downtime for the implementation of the new fleet project.

\subsection{Geology face mapping and modelling of veins}

A variety of geological applications can be performed using underground scan data. The use of underground LIDAR technology increases speed and accuracy, and helps bring field information into a digital format for different stakeholders. The direct digitation by LIDAR greatly improves the efficiency of the current standard workflow of manually recording information on paper, then transferring data to software for further analysis.

Geological field mapping is a usual practice that involves the collection of high detail structural measurements to obtain structural geology, rock mass characterisation, rock quality designation (RQD), lithological contacts, and other information in both active and inactive areas of the mine. Face mapping may also assist in the collection of helpful information to update geologic models, which are used to compare against block models. Accurate block models facilitate accurate and efficient production plans.

Geological mapping requires considerable visual observation and interpretation. The presence of the geologist in the field can be just as important as the highly detailed 3D representation of geological structures that results from LIDAR data. Detailed laser scans, complete with a high-resolution image capture, offer an immersive experience with 3D information in real coordinate systems. Geologists can easily interpret accurate descriptions of mineralogy, alterations, rock type, joints, faulting or foliation from the high-resolution scans and associated image. Features are quickly and easily digitised directly in 3D space to allow an accurate and timely product.

Standard resolution scans, complete in minutes, provide robust detail and allow the geologist to create a highly detailed and accurate picture of local geology. A single scan can cover an extent of approximately 20-25 linear metres, meaning two or three scans could cover a couple of rounds in the production area before ground control is in place without interfering with mine operations.

\subsection{Vein mapping}

The following example of drift ore model reconciliation was created in 25 minutes. Ten minutes was spent in the field collecting data, and 15 minutes of office time was required to process the data and create models.

Taking advantage of the intensity of data that the laser scan can collect at each point, the geologist identified the vein in the wall on both sides of the drift with spray paint. The optical intensity of the laser beam is represented with greyscale colours depending on different materials and colours in the rock surface (Figure 6(b)). In this case, the paint in the wall is easily recognised in the data to assist in the direct digitisation in 3D of the ore boundary in the wall (Figure (b)). 


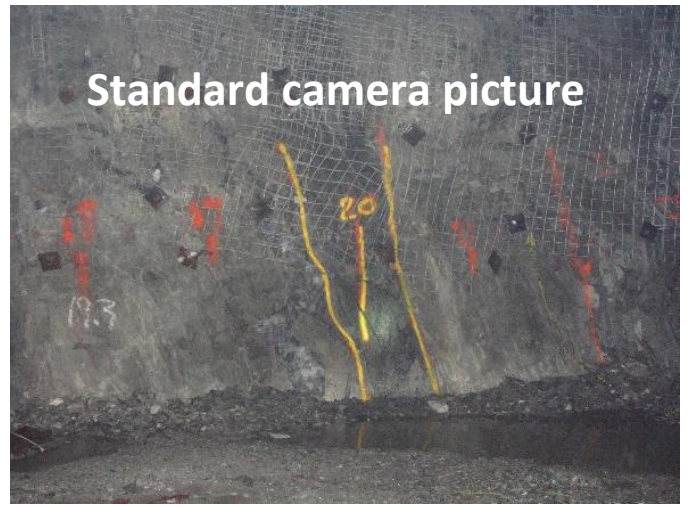

(a)

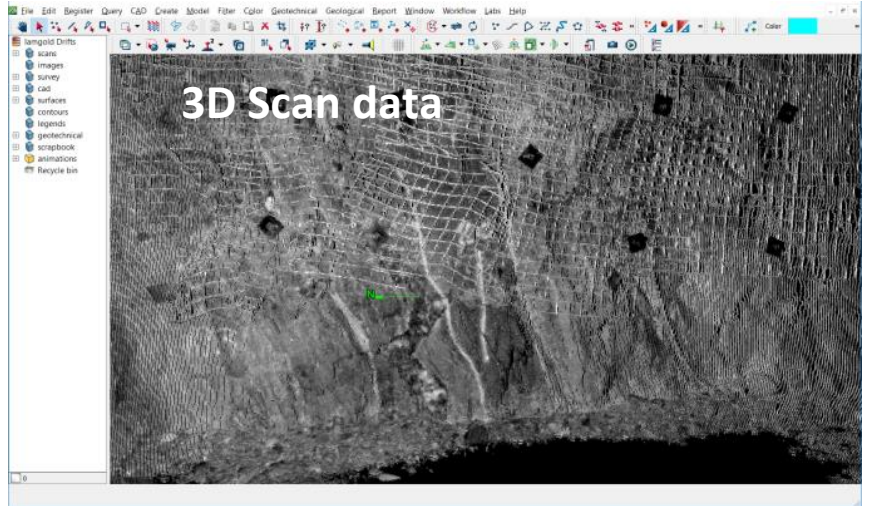

(b)

Figure 6 Comparison of the photo (a) with intensity data from the scan (b)

The boundary is drawn in 3D over the point cloud. Following the greyscale paint marks differentiated by the laser scan, the geologist digitised a polygon on the walls and roof of the drift. The geologist also utilised rock colour captured in the image, which differentiates the vein from the waste rock, when creating polygons. It is helpful that the points of the polygons be made at regular intervals to facilitate the modelling process afterwards. The result is two polygons that represent the ore-waste contacts along the walls and roof of the drift.

The geologist created two surfaces that represent ore-waste contacts (see red surfaces in Figure 7). The software can then create an automatic loop triangulation that represents vein thickness between the two boundary surfaces (see the green triangulation in Figure 7).

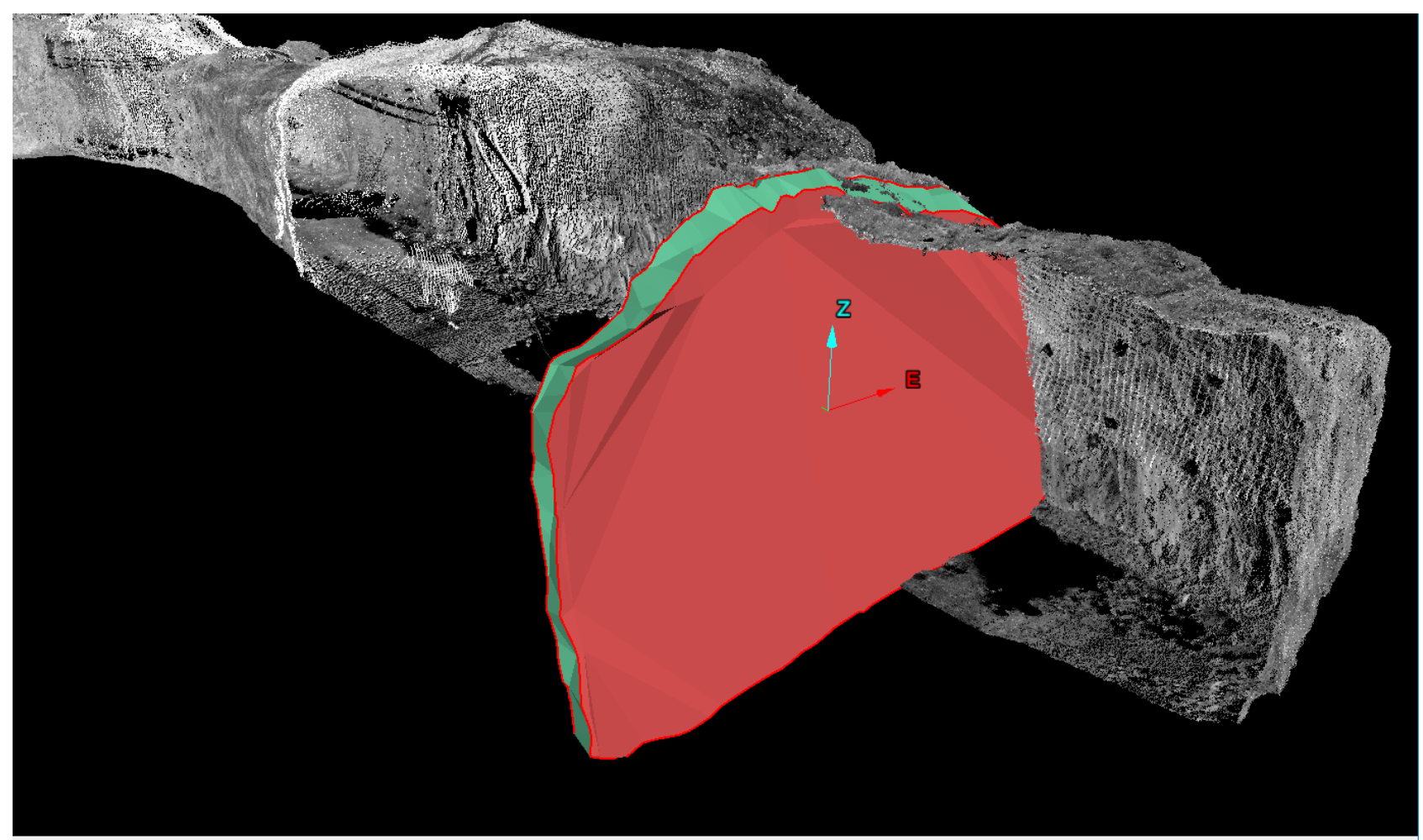

Figure 7 Vein solid extracted from the 3D mapping and modelling

The two red ore-waste triangulations and the green loop triangulation are combined to create a new validated solid, which can be used to calculate volume. 
Note that the polygons, as well as any other ore boundary CAD line that is drawn in a drift, can be used to create triangulations for model reconciliation.

The full workflow described is required on a weekly basis and, the quality of the data can assure a high level of precision in the ore model reconciliation. Using conventional technics, such as total station, requires double time and personnel in the field to come to only an approximation of the level of detail and accuracy that is obtained with the scan data.

\subsection{Conformance to design and drilling control}

The success of a mine design depends on its implementation. The ability to drill and blast to design specifications impacts the cost of the operation. One example of this is an oversize of the section of the drift where additional tonnages of material need to be extracted, therefore increasing the associated haulage cost. The presented example shows a $25 \mathrm{~m}$ tunnel in progress. Figure 8 shows a heat map of the actual surface coloured by distance against the design. The blue highlights underdug areas of $>50 \mathrm{~cm}$ distance from the design.
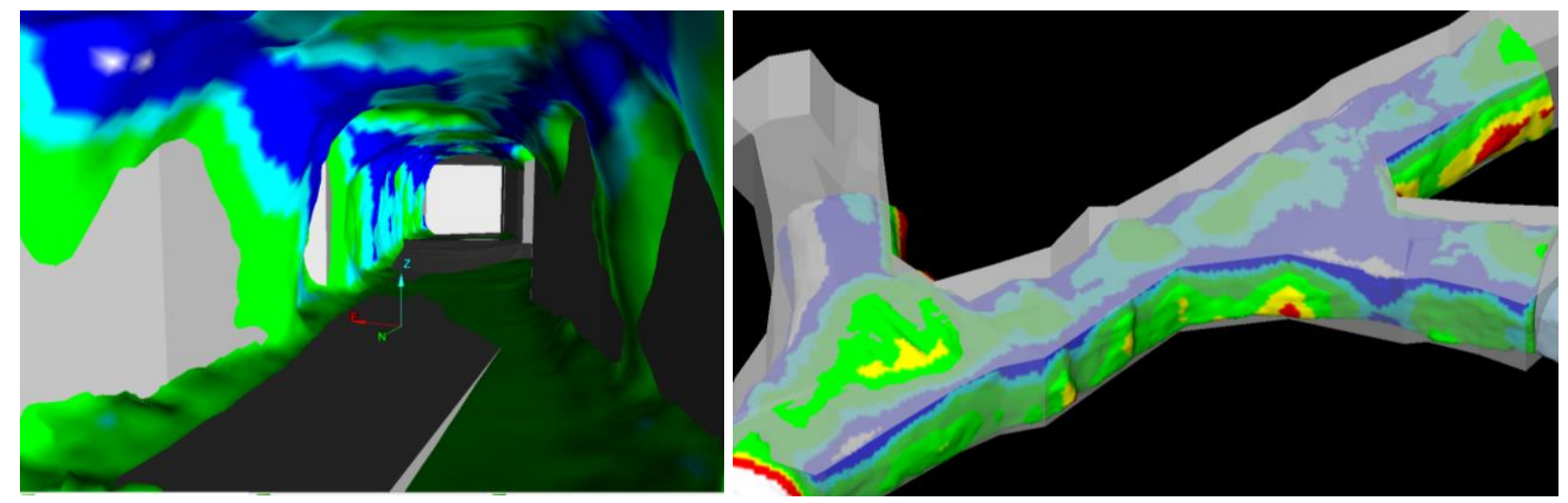

Figure 8 Heat map colour of the scanned surface showing underdug areas in light blue and blue

The initial purpose of this analysis was to detect any considerable areas of overbreak that affect overall excavation cost. Instead, an $8 \%$ underdig volume difference was identified. The discovery of this difference lead to the conclusion that the underdig may affect other processes, such as longhole drilling reconciliation.

During the stope drilling process, the toes of breakthrough holes are measured to help calculate deviation from the plan and adjust the remaining holes accordingly. In the current methodology, the coordinate of the hole is measured in $x-y$ and uses the flat roof from the design as a reference for the elevation coordinate. The difference in height between the designed roof and the actual rock roof produces an offset from the real position of the drillhole, which affects the angle of inclination and length. Using the traditional method, the adjustment of the remaining holes is calculated incorrectly.

In Figure 9, the yellow dotted line represents the actual location of the drillhole. The red line shows the current estimated position of the drillhole based on the vertical projection to the roof. The scan data represents the exact 3D representation of the surface, thus the identification of the longhole coordinates for an accurate interpretation and adjustment of the remaining holes. 

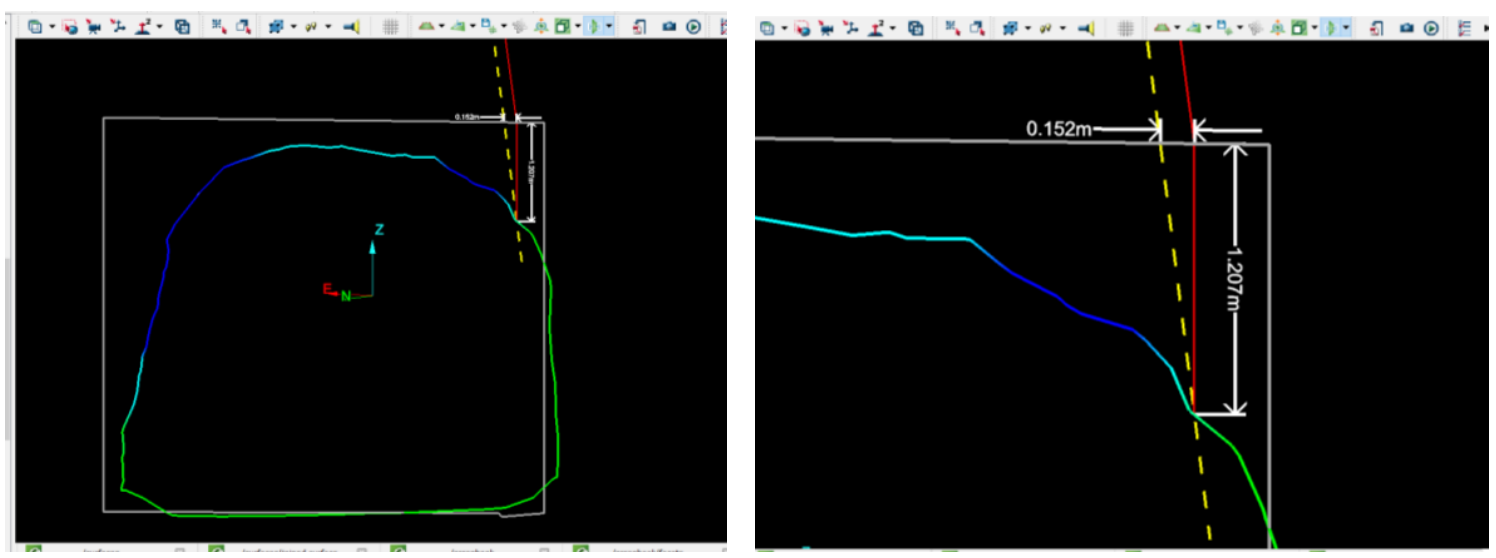

Figure 9 Section view. Horizontal and vertical offset distance between estimated and actual position of the drillhole

Another important benefit was the speed to determine the coordinates of the holes so the driller does not need to wait too long to make the adjustment. With the scan data registered within minutes, the feedback to the drillers was delivered in less than 30 minutes, which is three times faster than the current process.

\subsection{Alimak scanning}

The following example shows the work done to scan a raise built out of an Alimak. The raise was $205 \mathrm{~m}$ long with an inclination of $82^{\circ}$. The purpose of the measurement was to obtain an accurate volume, accurate geometry and a detailed shape to identify accurate intersections on the upper levels.

Two scans were made on the drift and registered using a total station that gave precise coordinates to the scanner location and backsight. These two scans were used as a fixed reference data for the next scans. A third free scan was made at the base of the raise, collecting data towards the drift and up the raise. Using the initial positioning and global registration tools, the free scan shown in red in Figure 10 was registered against the previous two fixed scans.

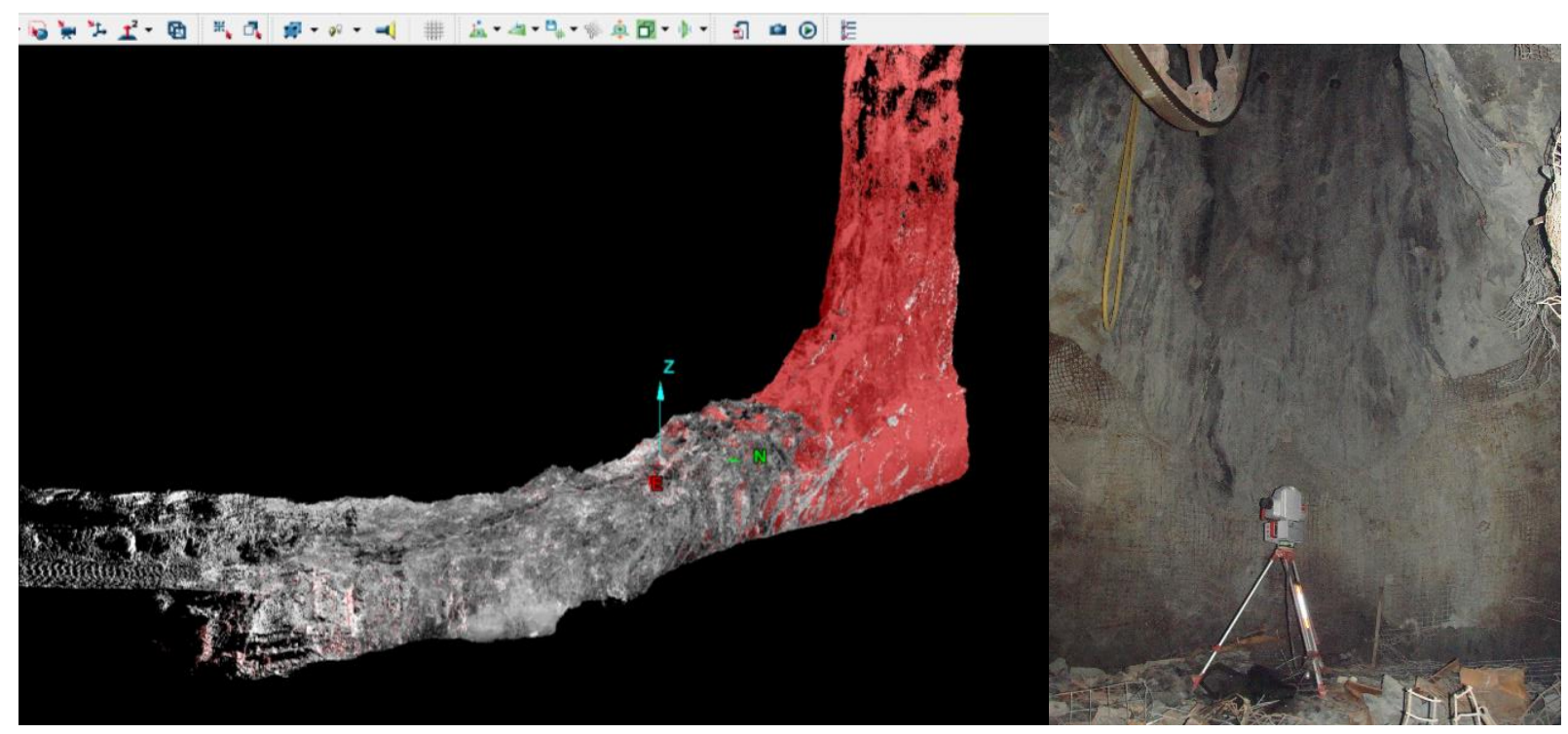

Figure 10 Fixed reference scans in grey and free scan in red. On the right is the scanner at the base of the raise

To scan the raise, it was necessary to attach the scanner to the roof of the Alimak, as shown in Figure 11. This was done to make it possible to make consecutive scans in a stop-and-go mode while moving upwards. Long bolts, plus a special plate that acted as a clamp, hold the scanner firmly against the Alimak structure. 


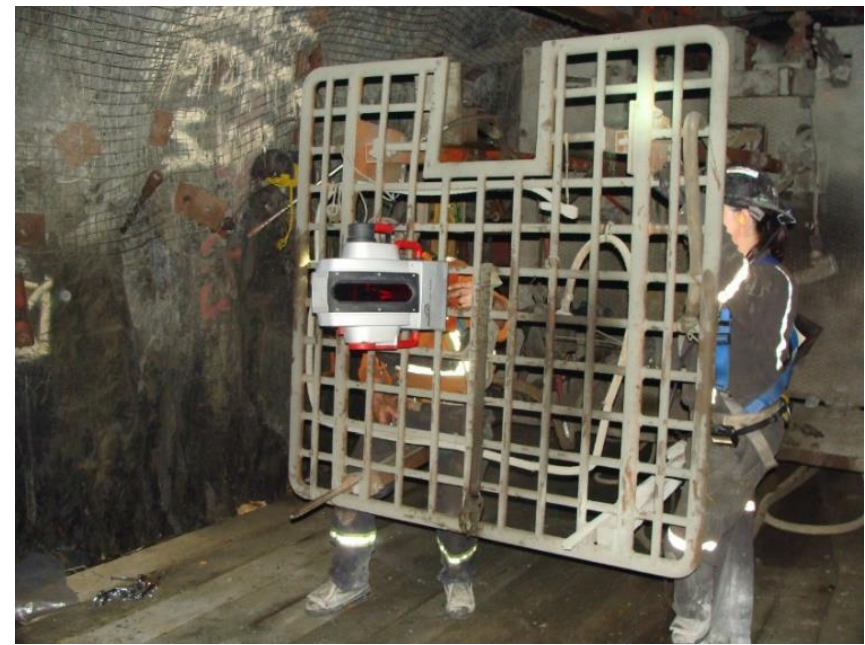

Figure 11 Scanner unit attached to the Alimak

The first scan from the Alimak was approximately $10 \mathrm{~m}$ into the raise in a nearly vertical orientation. The data was collected via a wireless connection between the scanner and the tablet. After a scan completed, digital data was checked to ensure the scan successfully completed.

The Alimak was moved up the raise an additional $10 \mathrm{~m}$, and a new scan was made. The distance was expected to be appropriate to ensure proper overlap with the previous scan for the registration process. The same process was repeated 13 times until reaching approximately $150 \mathrm{~m}$ over the ground.

Each $10 \mathrm{~m}$ scan of the raise took three minutes. The last scan covered most of the last $50 \mathrm{~m}$. The entire scanning process, including the initial drift scans and $200 \mathrm{~m}$ of raise, was complete in two hours and 20 minutes (Figure 12(a)). The processing time to register the scans and generate a model was approximately one hour.

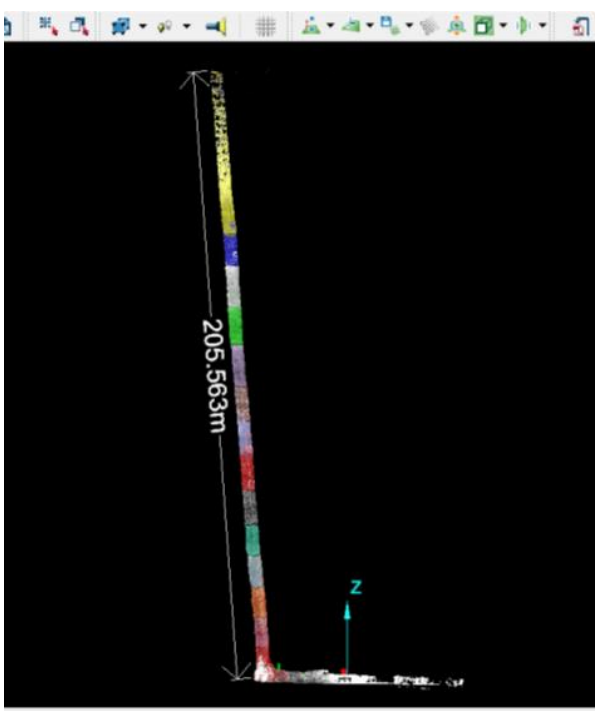

(a)

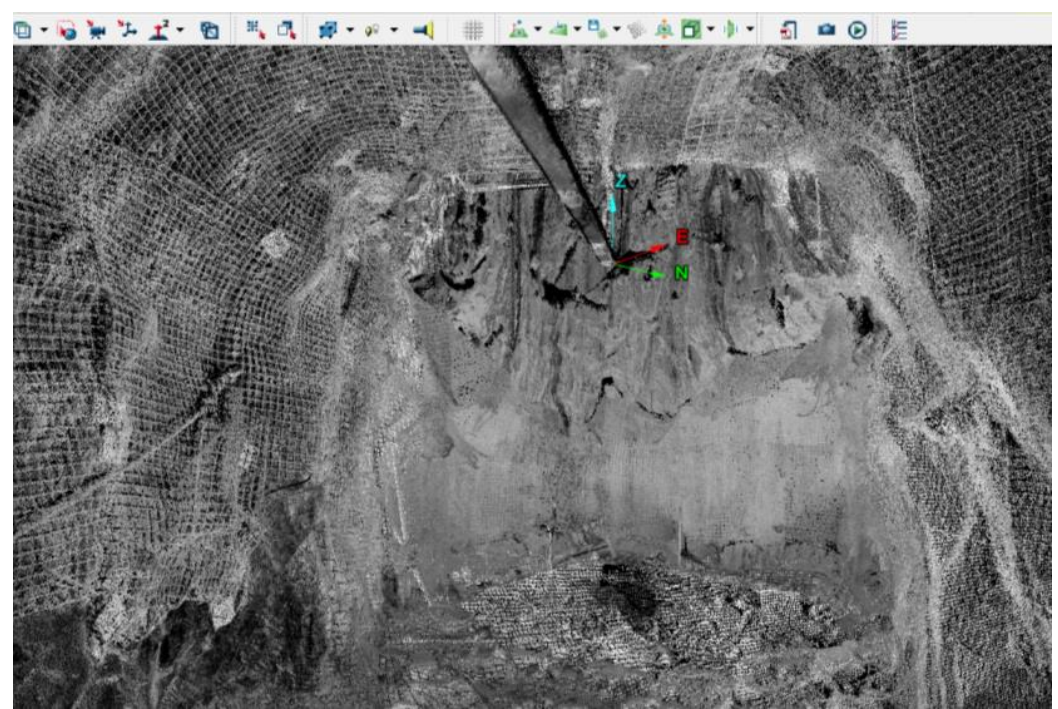

(b)

Figure 12 (a) All the scans together indicating the length of the raise; and, (b) The inside view of the drift and base of the raise. The rail of the Alimak is seen at the top

The scanning solution proved to be a powerful tool to scan in a difficult environment such as the Alimak raise. The prior methodology measured isolated points with a total station, however the level of detail is not enough to generate a model and calculate volumes and/or determine dilution. The new methods yield not only volume information, but include additional information which allows for analysis of rail alignment and detailed 3D locations of existing bolts which provide ground support along the raise. 


\section{Conclusion}

As it has been presented, laser-derived point cloud data is a powerful tool for a variety of underground applications. The possibility of collecting and processing data in short periods of time can reduce considerably the gap between the field and the office, improving quantity and quality of data. The examples outlined in this paper show the value of speed, accuracy and safety. Cost reduction can be obtained by controlling key factors such as overdig and underdig applications (haulage cost optimisation), drillhole deviation control, and faster adjustment time of the driller (reducing driller downtime per round and accelerating the drill and blast process). Alimak raise application has shown a different approach to collect highly detailed information from inaccessible areas in a safe manner. The examples also demonstrate how visualisation in a 3D environment helps facilitate understanding and communicates the outputs in an easy way to the stakeholders in the mine.

The acquisition of 3D information is a starting point for digital databases, which are part of a process of continuous improvement. The present examples have shown some key elements for engineers and geologists to improve the decision-making process and optimise operational assets. 
